
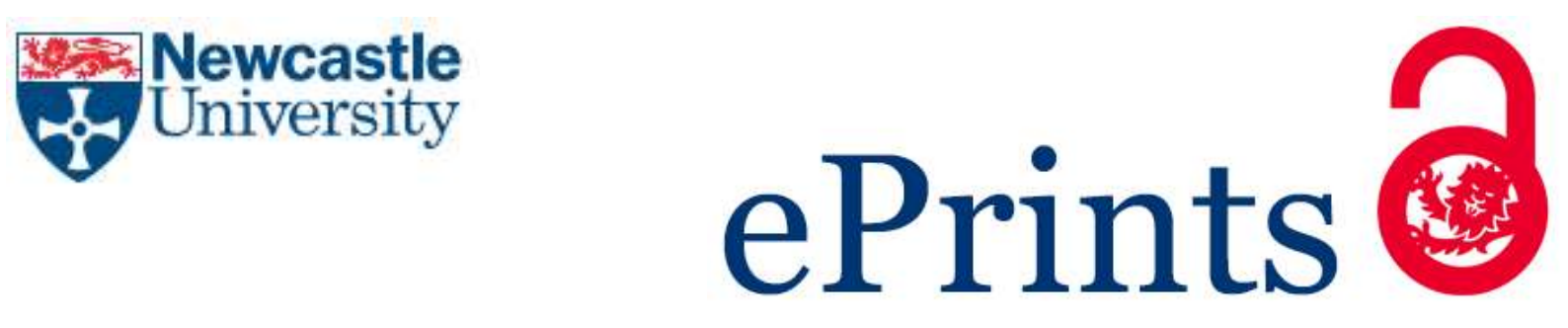

Klein M, Kasten C, Chakraborty N. A-priori Direct Numerical simulation assessment of models for generalized sub-grid scale turbulent kinetic energy in turbulent premixed flames. Computers and Fluids 2017, 154, 123-131. Copyright:

(C) 2017. This manuscript version is made available under the CC-BY-NC-ND 4.0 license

DOI link to article:

https://doi.org/10.1016/j.compfluid.2017.05.028

Date deposited:

$21 / 06 / 2017$

Embargo release date:

30 May 2018

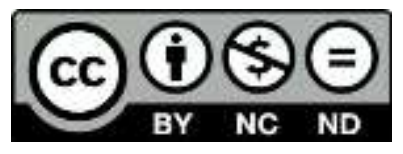

This work is licensed under a

Creative Commons Attribution-NonCommercial-NoDerivatives 4.0 International licence 


\title{
A-priori Direct Numerical simulation assessment of models for generalized sub-grid scale turbulent kinetic energy in turbulent premixed flames
}

\author{
M. Klein ${ }^{1 *}$, C. Kasten ${ }^{1}$, N. Chakraborty ${ }^{2}$ \\ ${ }^{1}$ Universität der Bundeswehr München, \\ Fakultät für Luft- und Raumfahrttechnik, LRT1, \\ Werner-Heisenberg-Weg 39, 85577 Neubiberg, Germany \\ Email: markus.klein@unibw.de Phone:+49(0)8960042122 \\ ${ }^{2}$ School of Mechanical and Systems Engineering \\ University of Newcastle \\ Claremont Road, Newcastle \\ NE1 7RU, UK \\ Email: nilanjan.chakraboty@ncl.ac.uk Phone: +44(0)1912083570
}

\section{HIGHLIGHTS}

- Demonstration of flame induced anisotropy using the Lumley triangle

- Demonstration of the complex interaction of numerical scheme and physical models

- An alternative formulation of Clarks model for evaluating Sub-grid kinetic energy

- Identification of a suitable model constant for Yoshizawa's model

- Demonstration of Lewis number and heat release parameter effects on sub-grid stresses

\footnotetext{
${ }^{*}$ Corresponding author
} 


\section{ABSTRACT}

The fidelity of Large Eddy Simulation (LES) in the context of turbulent premixed combustion modelling depends on the complex coupling between turbulence and chemical reactions occurring at the unresolved scale. Although LES of combustion systems is becoming increasingly popular, the closures for sub-grid scale (SGS) stresses have mostly been derived assuming constant density flows. Similar to the unclosed scalar flux, the behaviour of the SGS stresses depends on the balance between heat release and turbulence, and it has been shown recently that counter-gradient transport (CGT) can occur for the stress tensor when the isotropic part of the stress tensor is not properly accounted for. This leads to a negative correlation between the predictions obtained from an eddy viscosity type model and the stresses obtained from Direct Numerical Simulation (DNS). In the present work the modelling of the isotropic part of the stress tensor, closely related to the generalised sub-grid scale kinetic energy, is considered in detail. To this end the interplay between SGS dilatation effects and unresolved velocity fluctuations is analysed using a-priori DNS analysis of turbulent, statistically planar flames with different values of global Lewis number and heat release parameter. Well-known models for generalised sub-grid scale kinetic energy have been assessed in the context of turbulent premixed combustion and detailed physical explanations for their behaviour have been provided. Further, the effects of SGS dilatation rate on the anisotropy of the SGS stresses have been highlighted using a variant of the Lumley triangle.

Keywords: Generalised sub-grid scale kinetic energy, sub-grid stresses, Large Eddy simulations, Direct Numerical Simulations, turbulent premixed combustion 


\section{INTRODUCTION}

Large Eddy Simulation (LES) is considered to be a promising technique for flows featuring unsteady, large coherent structures. Despite increasing popularity of combustion LES, the closures for sub-grid scale (SGS) stresses for constant density flows are usually used although they do not adequately capture the gas-dynamic expansion in turbulent premixed flames [1,2]. The filtered momentum conservation equation is given as:

$$
\frac{\partial \bar{\rho} \widetilde{u}_{l}}{\partial t}+\frac{\partial\left(\bar{\rho} \widetilde{u_{\imath}} \widetilde{u}_{\jmath}\right)}{\partial x_{j}}=-\frac{\partial\left(\overline{\rho u_{l} u_{\jmath}}-\bar{\rho} \widetilde{u_{l}} \widetilde{u_{\jmath}}\right)}{\partial x_{j}}+\frac{\partial}{\partial x_{j}} \bar{\rho} \tilde{v}\left(\left(\frac{\partial \widetilde{u_{j}}}{\partial x_{i}}+\frac{\partial \widetilde{u_{l}}}{\partial x_{j}}\right)-\frac{2}{3} \frac{\partial \widetilde{u_{k}}}{\partial x_{k}} \delta_{i j}\right)-\frac{\partial \bar{p}}{\partial x_{i}}
$$

where $\rho, u_{i}$ and $v$ denote the gas density, $i^{\text {th }}$ component of velocity vector and kinematic viscosity respectively. The filtering operation is given by: $\overline{Q(\boldsymbol{x})}=\int Q(\boldsymbol{x}-\boldsymbol{r}) G(\boldsymbol{r}) \boldsymbol{d} \boldsymbol{r}$, where $G(\boldsymbol{r})=\left(6 / \pi \Delta^{2}\right)^{3 / 2} \exp \left(-6 \boldsymbol{r} \cdot \boldsymbol{r} / \Delta^{2}\right)$ is the Gaussian filter kernel, $\Delta$ is the filter width and $\widetilde{Q}=\overline{Q \rho} / \bar{\rho}$ denotes Favre filtering. The SGS stress tensor is given by $\tau_{i j}^{S G S}=\overline{\rho u_{\imath} u_{J}}-\bar{\rho} \widetilde{u_{\imath}} \widetilde{u}_{J}$ [3], and the isotropic part of the SGS stresses, i.e. the term involving $-\frac{1}{3} \tau_{k k}^{S G S} \delta_{i j}$, is often added to the filtered pressure for incompressible flows. The relevance of modelling the isotropic part of the SGS stresses is discussed in some detail in reference [4]. The most popular closure for $\tau_{i j}^{S G S}$ is the static Smagorinsky model (SSM), which is given below where

$$
\begin{aligned}
& \widetilde{S_{l j}}=\left(\partial \widetilde{u_{l}} / \partial x_{j}+\partial \widetilde{u_{j}} / \partial x_{i}\right) / 2 \text { and }\left|\widetilde{S_{l \jmath}}\right|=\sqrt{2 \widetilde{S_{l j}} \widetilde{S_{l j}}} \text { : } \\
& \tau_{i j}^{S S M}=-2 \bar{\rho}\left(C_{s} \Delta\right)^{2}\left|\widetilde{S_{l j}}\right|\left(\widetilde{S_{l \jmath}}-\widetilde{S_{k k}} \delta_{\mathrm{ij}} / 3\right)
\end{aligned}
$$

The constant $C_{s}$ is either set to $C_{s} \approx 0.18$ in the static model version (SSM) or can be determined in a dynamic manner (see Ref. [3] for details). Pfadler et al. [2] demonstrated the Smagorinsky model's unsatisfactory performance based on direct measurements of the density-weighted stress tensor. Klein et al. [4] recently showed that counter-gradient transport (CGT) for the stress tensor 
is obtained if the isotropic part of the stress tensor is not properly accounted for. The behaviour of the SGS stresses depends on the balance between heat release and turbulence [4] and the competition between these effects will be analysed in this work by means of a-priori DNS analysis of statistically planar flames with different global Lewis numbers $L e$ (i.e. ratio of thermal to mass diffusivities) and heat release parameters $\tau=\left(T_{a d}-T_{0}\right) / T_{0}$ where $T_{a d}$ and $T_{0}$ are the adiabatic flame and unburned gas temperatures respectively.

An explicit model (i.e. YOS model) for the isotropic part of the SGS stress tensor has been suggested by Yoshizawa [5]: $\tau_{k k}^{Y O S}=2 C_{I} \bar{\rho} \Delta^{2}\left|\widetilde{S_{l l}}\right|^{2}$. Yoshizawa [5] recommended a value of $C_{I} \approx$ 0.089 whereas Moin et al. [6] reported a value of $C_{I}$ ranging from 0.0025 to 0.009 . This spread of $C_{I}$ arises to some extent due to the fact that some studies used a mixed model where the scalesimilarity part has a contribution to the diagonal SGS stress components [7]. All these aforementioned studies have been conducted in the context of non-reacting flows. Klein et al. [4] demonstrated that satisfactory results could only be obtained for the dynamic evaluation of $C_{I}$ using the formula $C_{I}=\left\langle L_{k k} M\right\rangle /\left\langle M^{2}\right\rangle$ instead of $C_{I}=\left\langle L_{k k}\right\rangle /\langle M\rangle$ [6], in combination with an averaging conditional on $\tilde{c}$. Here, $M$ is defined as: $M=2 \hat{\bar{\rho}} \widehat{\Delta}^{2}\left|\widehat{S_{l \jmath}}\right|^{2}-\left.2 \bar{\rho} \widehat{\Delta^{2} \mid \widetilde{S_{l J}}}\right|^{2}$ and $L_{k k}=$ $\widetilde{\tilde{u}_{k} \tilde{u}_{k}}-\widehat{\widetilde{u}_{k}} \widehat{\widetilde{u}}_{k}$ is the Leonard stress [6]. Depending on the turbulence intensity and the LES filter width, values of $C_{I}$ in the range 0.1-0.2 were found within the flame brush [4], which is considerably larger than the values for isothermal flows reported in the literature. In this work $C_{I}$ will be globally determined based on a least-squares fit between the modelled and exact stresses obtained from DNS data. Although several analyses (Refs. [8,9] and references therein) deal with the LES modelling of the SGS scalar fluxes, limited effort has been directed to the assessment of 
SGS stress tensor closures in turbulent premixed combustion [2], and in particular to the modelling of the isotropic part of the stress tensor, which is the focus of this work. The quantity $\tau_{k k}^{S G S}$ is closely related to the generalized SGS kinetic energy formally introduced by Germano in the context of constant density flows as [10]: $k^{g s g s}=\frac{1}{2} \tau_{k k}^{S G S} / \bar{\rho}$. For completeness, it is worth mentioning that $k^{g s g s}$ is equal to the SGS kinetic energy $k^{s g s}=\frac{1}{2} \overline{u_{\Delta l}^{\prime} u_{\Delta l}^{\prime}}\left(\right.$ where $\left.u_{\Delta i}^{\prime}=u_{i}-\bar{u}_{\imath}\right)$, if the filter is a Reynolds operator, which is generally not the case [3]. Besides the modelling of the isotropic part of the stress tensor, $k^{g s g s}$ is also used in different contexts. The velocity fluctuation $u_{\Delta}^{\prime}=\sqrt{2 k^{g s g s} / 3}$ represents an important input to many combustion models. Exemplarily, it determines the turbulent flame speed in the G-equation approach [11], it enters the efficiency function in the artificially thickened flame approach [12] and it also effects the wrinkling factor in Flame Surface Density based modelling [13,14]. Furthermore $k^{g s g s}$ is often used to assess the degree of resolution of a LES simulation $[15,16]$. The main objectives of the present analysis are to: (a) assess existing models for $\tau_{k k}^{S G S}$ based on a-priori analysis of DNS data for statistically planar turbulent flames with different $L e$ and $\tau$, and (b) provide detailed explanations for the observed behaviour.

The rest of the paper will be organized as follows. The information pertaining to mathematical background and numerical implementation will be provided in the next section. This will be followed by the presentation of results and their discussion. The main findings will be summarized and conclusions will be drawn in the final section of this paper.

\section{MATHEMATICAL BACKGROUND \& NUMERICAL IMPLEMENTATION}


The DNS database considered here was used previously in several studies [8,9,17-23]. It consists of five statistically planar flames with $L e=0.34$ (case A), 0.6 (case B), 0.8 (case C), 1.0 (case D) and 1.2 (case $\mathrm{E}$ ). The heat release parameter $\tau=\left(T_{a d}-T_{0}\right) / T_{0}$ for these cases is taken to be 4.5. An additional case (case F) is considered to analyse the effects of $\tau$, where $\tau$ has been set to $\tau=3.0$ and $L e$ is taken to be 1.0. Standard values of Prandtl number $\operatorname{Pr}=0.7$, Zel'dovich number $\beta_{Z}=$ 6.0 and ratio of specific heats $\gamma_{g}=1.4$ were considered for the present analysis. The initial values of the ratio of the root-mean-square turbulent velocity fluctuation and unstrained laminar burning velocity $u^{\prime} / S_{L}$ and the integral length scale to thermal flame thickness ratio $l / \delta_{t h}$ are taken to be 7.5 and 2.5 respectively (i.e. $u^{\prime} / S_{L}=7.5$ and $\left.l / \delta_{t h}=2.5\right)$ in all cases, where $\delta_{t h}=\left(T_{a d}-\right.$ $\left.T_{0}\right) / \max |\nabla \hat{T}|_{L}$ is the thermal flame thickness. The initial values of Damköhler $D a=l S_{L} / u^{\prime} \delta_{t h}$ and Karlovitz $K a=\left(u^{\prime} / S_{L}\right)^{3 / 2}\left(l / \delta_{t h}\right)^{-1 / 2}$ numbers are 0.33 and 13.0 respectively. All cases considered here represent the thin reaction zones regime [24] combustion.

The values of $u^{\prime} / S_{L}$ and $l / \delta_{t h}$ used here remain comparable to several previous analyses [25-29] for a-priori DNS modelling. Furthermore, the models proposed based on a-priori DNS analyses using this database $[22,23]$ have been found to be in good agreement with a-posteriori assessments based on actual LES simulations [30,31]. It is worth noting that the findings of this paper are also valid for the database used in Ref. [4] which deals with different values of turbulent Reynolds number $R e_{t}$. Furthermore, it is discussed in the Appendix A1 that all findings reported in this paper remain both qualitatively and quantitatively valid for an additional unity Lewis number case with considerably higher values of $R e_{t}$ and scale separation $l / \delta_{t h}$. This provides the confidence in the findings of the present analysis which has been conducted for a moderate dynamic range in favour of an extensive parametric study in terms of Lewis number, heat release parameter, and filter width. 
The physical mechanisms responsible for the competing effects of heat release and turbulence on $\tau_{k k}^{g s g s}$ can be captured using simplified chemistry. Therefore a single step irreversible chemical mechanism has been considered here. For all cases the simulation domain size was taken to be $24.1 \delta_{t h} \times 24.1 \delta_{t h} \times 24.1 \delta_{t h}$, which was discretised using a uniform Cartesian mesh of $230^{3}$ grid points. High order finite-difference $\left(10^{\text {th }}\right.$ order for the internal grid points and one-sided $2^{\text {nd }}$ order scheme at non-periodic boundaries) and $3^{\text {rd }}$ order low storage Runge-Kutta schemes are used for spatial differentiation and explicit time advancement respectively. The boundary conditions in the mean flame propagation direction (aligned with negative $x_{1}$-direction) are taken to be partially non-reflecting, whereas boundaries in transverse directions are taken to be periodic.

The turbulent velocity fluctuations are initialised using a homogeneous isotropic incompressible velocity field. The reacting flow field is initialised by a steady planar unstrained premixed laminar flame solution. In all cases flame-turbulence interaction takes place under decaying turbulence and all non-dimensional numbers mentioned before have to be understood as initial values here and in the remainder of the text. By the time statistics were extracted the value of $u^{\prime} / S_{L}$ in the unburned gas ahead of the flame decayed by about $50 \%$ of its initial value, whereas the value of $l / \delta_{t h}$ in the fresh gas increased by about 1.7 times. The simulation time $t_{s i m}=3.34 l / u^{\prime} \approx \delta_{t h} / S_{L}$ is comparable to several previous DNS studies which focused on the modelling of turbulent premixed combustion $[25,27-29,32,33]$. The data is taken from a single frame where turbulent kinetic energy and the global burning rate were not changing rapidly with time as shown in [11]. Moreover, it was shown there that the results remain qualitatively similar halfway through the simulation. 
For this analysis, the DNS data has been explicitly filtered using a Gaussian filter kernel. Results will be presented from $\Delta \approx 0.4 \delta_{t h}$ where the flame is almost resolved, up to $\Delta \approx 2.8 \delta_{t h}$ where the flame becomes fully unresolved and $\Delta$ is comparable to the integral length scale $l$.

\section{CLOSURES FOR GENERALISED SGS KINETIC ENERGY}

It was shown elsewhere $[4,8,9]$ that Clark's tensorial model [34], henceforth denoted as the CTM model, satisfactorily represents SGS quantities in turbulent premixed flames. Applying this model to the isotropic part of the stress tensor yields:

$$
\tau_{k k}^{C T M}=\bar{\rho}\left(\Delta^{2} / 12\right)\left(\partial \widetilde{u_{k}} / \partial x_{i}\right)\left(\partial \widetilde{u_{k}} / \partial x_{i}\right)
$$

By including density effects Vreman [35] proposed the following expression, henceforth denoted as the DSS (i.e. density based scale-similarity) model:

$$
\tau_{k k}^{D S S}=C_{D S S}\left(\overline{\bar{\rho} \widetilde{u_{k}} \widetilde{u_{k}}}-\overline{\bar{\rho} \widetilde{u_{k}}} \overline{\bar{\rho} \widetilde{u_{k}}} / \overline{\bar{\rho}}\right)
$$

The model coefficient $C_{D S S}$ depends on the primary and secondary filter widths. For the secondary filter used in this work, $C_{D S S}=0.5$ yields results consistent with the other modelling approaches.

There are similarities between the YOS, CTM and DSS models. Expanding the term $\left|\widetilde{S_{l \jmath}}\right|^{2}=$ $2 \widetilde{S_{l j}} \widetilde{S_{l j}}$ in the YOS model gives three terms of the form $2\left(\partial u_{i} / \partial x_{i}\right)^{2}, i=j$, further six terms of the form $\left(\partial u_{i} / \partial x_{j}\right)^{2}, i \neq j$ and finally three mixed gradients $2\left(\partial u_{i} / \partial x_{j}\right)\left(\partial u_{j} / \partial x_{i}\right)$. Comparing this with Eq. 3 shows that nine out of the 12 terms contained in the YOS model are identically the same in the CTM model, if one excludes the pre-factor 2 in $2\left(\partial u_{i} / \partial x_{i}\right)^{2}, i=j$. This similarity between the CTM model (which is of scale-similarity type) and the YOS model (which is of entirely different nature) is also reflected in the correlation coefficients and will be discussed in 
the next section. Neglecting the gradient products of mixed type in the YOS model (i.e. three out of the twelve terms) and comparing its terms to the CTM model provides an opportunity to come up with an estimated model coefficient for $C_{I}$ in the range of $C_{I}=(1 / 48)-(1 / 24)$ depending on whether more weight is given to $\left(\partial u_{i} / \partial x_{j}\right)^{2}, i \neq j$ or to $2\left(\partial u_{i} / \partial x_{i}\right)^{2}, i=j$. It is interesting to note that these $C_{I}$ values are (considerably) larger than the ones suggested in Refs. [5-7]. As a starting point the parameter $C_{I}=1 / 24$ has been chosen.

Noting that $k^{g s g s}=\frac{1}{2} \tau_{k k}^{S G S} / \bar{\rho}$, the realizability analysis by Vremen et al. [36] yields: $C_{I} \geq$ $\frac{1}{2} \sqrt{3} C_{s}^{2}$ in the context of the present work. Using the theoretical, isotropic turbulence value for the Smagorinsky constant $C_{s}=0.18$ results in the realizability condition $C_{I} \geq 0.0281$. Hence, realizability is clearly fulfilled for $C_{I}=\frac{1}{24}=0.0417$, and thus satisfies the realizability conditions that were derived in [36]. Note, that in practice the value of $C_{s}$ is often adjusted to lower values e.g. $C_{s}=0.1$ for channel flows, in fact $C_{s}=0.092$ is mentioned in [36]. This would result in the lower bound $C_{I} \geq 0.0073$.

Throughout this work $\bar{Q}_{i, j, k}=\sum_{\hat{\imath} \hat{\jmath}=-1,1} a_{\hat{\imath}} a_{\hat{\jmath}} a_{\hat{k}} Q_{i+\hat{\imath}, j+\hat{\jmath}, k+\hat{k}}$ is used [37], where $\left(a_{d-1}, a_{d 0}, a_{d 1}\right)=$ $(C, 1-2 C, C)$ with $0<C \leq 1 / 3$ being a free parameter. A typical value corresponds to $C=$ $1 / 12$ according to the suggestion of Ref. [37] where the corresponding transfer function is given. As an alternative one can consider a Laplacian filter given by: $\bar{Q}_{i, j, k}=Q_{i, j, k}+C \Delta^{2} \nabla \cdot \nabla\left(Q_{i, j, k}\right)$ where $\nabla \cdot \nabla$ is a discrete finite-difference expression for the Laplace operator. A value of $C=$ $1 / 24$ yields $4^{\text {th }}$-order accuracy for a Gaussian filter [3]. The CTM model is commonly derived [3] by inverting $\bar{Q}_{i, j, k}=Q_{i, j, k}+C \Delta^{2} \nabla \cdot \nabla\left(Q_{i, j, k}\right)$ and making use of the product rule of differentiation. Alternatively, the CTM model can be written as $\tau_{k k}^{C T M *}=$ $\bar{\rho}\left(\Delta^{2} / 24\right)\left(\partial^{2}{\widetilde{u_{k}}}^{2} / \partial x_{i}^{2}-2 \widetilde{u_{k}} \partial^{2} \widetilde{u_{k}} / \partial x_{i}^{2}\right)$. This expression and Eq. 3 are equivalent but their 
finite-difference approximations are different. Unless mentioned otherwise, filtering operations or finite-differences in the present a-priori analysis have to be understood as sampling on the equivalent LES grid [38]. The positive semi-definiteness of the discretized form of $\tau_{k k}^{C T M *}$ is discussed in Appendix A2.

\section{RESULTS \& DISCUSSION}

The imbalance between conductive heat and diffusive-mass fluxes gives rise to an increase in burning rate and flame wrinkling in $L e<1$ flames in comparison to the $L e=1.0$ flame under similar unburned gas turbulence (see Refs. [8,19,39] and references therein), which can be substantiated from a comparison of the instantaneous views of $c$ isosurfaces for cases $\mathrm{A}$ and $\mathrm{D}$ in Fig. 1. Note, that in the context of simple chemistry, the reaction progress variable $c$ is defined in terms of a reactant mass fraction $Y_{R}$ as: $c=\left(Y_{R 0}-Y_{R}\right) /\left(Y_{R 0}-Y_{R \infty}\right)$ where subscripts 0 and $\infty$ are used to refer to the values in the unburned and fully burned gases respectively.
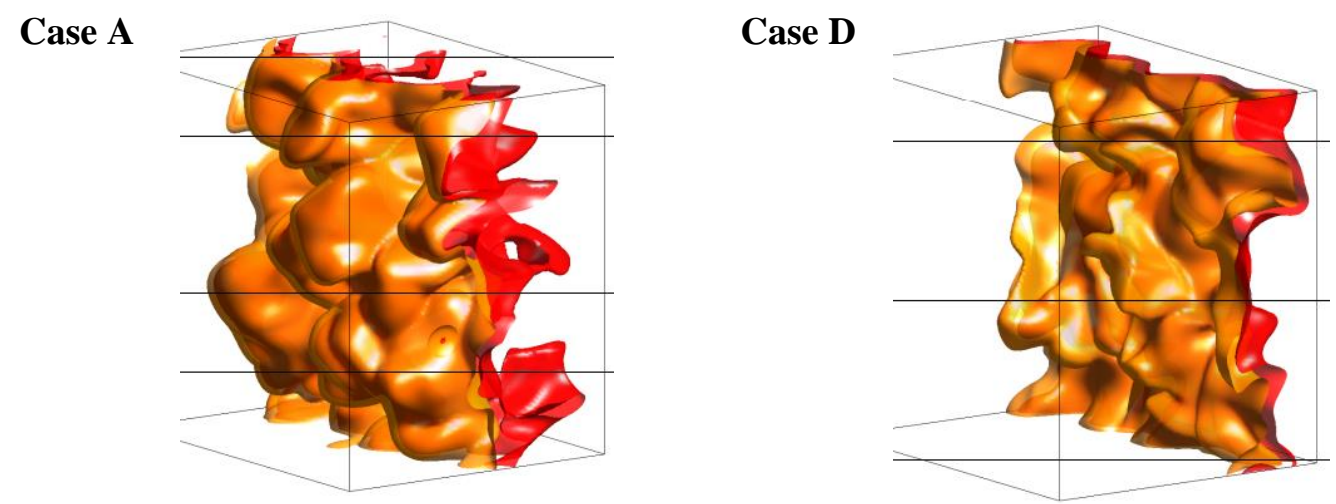

Figure 1: Instantaneous view of $c$ isosurfaces for cases A and D at $t=\delta_{t h} / S_{L}$. The value of $c$ increases from 0.1 (yellow) to 0.9 (red).

The competition between turbulent velocity fluctuations and flame normal acceleration determines the statistical behaviour of the SGS stresses: when the effects of flame normal acceleration are 
stronger than the effects of turbulent velocity fluctuation a counter gradient transport (CGT) is observed and vice versa [4]. Hence, for this database, predominant CGT is expected in cases A and B and relatively more GT is expected for example for cases E and F because flame normal acceleration is relatively weak in these cases due to smaller $\tau$ (for case $\mathrm{F}$ ) and higher $L e$ (for case E). The relation between Lewis number and flame normal acceleration can be understood in the following manner. The reactants diffuse into the reaction zone at a faster rate than the rate of thermal diffusion out of it in the case of $L e<1$, which in turn leads to simultaneous focusing of reactants and defocusing of heat in the positively stretched zones in turbulent $L e<1$ flames. This gives rise to simultaneous occurrence of high reactant concentration and temperature in positively stretched zones in turbulent $L e<1$ flames, which augments the rate of burning, magnitude of dilatation rate and thereby strengthens the flame normal acceleration in comparison to unity Lewis number flames with statistically similar unburned gas turbulence (see e.g. [18] and references therein). By contrast, thermal diffusion rate supersedes the mass diffusion rate in $L e>1$ flames and thus a combination of simultaneous strong defocusing of heat and weak focusing of reactants in the positively stretched zones leads to the weakening of the rate of burning, magnitude of dilatation rate and flame normal acceleration in comparison to unity Lewis number flames with statistically similar unburned gas turbulence. The enhancement of the rate of burning with decreasing Le can be substantiated from the fact that the volume-integrated reaction rate in the turbulent case normalised by the corresponding laminar value for the current $L e=$ $0.34,0.6,0.8,1.0,1.2$ cases are given by $13.70,4.58,2.53,1.83$ and 1.50 respectively [8] when the statistics were extracted. 
The mean values of the cosines of the angle $\Theta$ between SGS stresses $\left(\tau_{1 j}^{S G S}\right)_{j=1,2,3}$ evaluated from DNS and $\left(\tau_{1 j}^{S S M}\right)_{j=1,2,3}$ conditional on $\tilde{c}$ values for cases A, D, E and F for $\Delta / \delta_{t h}=0.4,1.6$ and 2.8 are shown in Fig. 2 (i.e. $\cos (\Theta)=\tau_{1 j}^{S G S} \tau_{1 j}^{S S M} /\left(\sqrt{\tau_{1 j}^{S G S} \tau_{1 j}^{S G S}} \sqrt{\tau_{1 j}^{S S M} \tau_{1 j}^{S S M}}\right)$ with summation over repeated indices). An angle $\Theta$ of $0^{0}\left(180^{\circ}\right)$ is associated with perfect GT (CGT) because the cosine assumes a value of $1.0(-1.0)$. Figure 2 shows that the amount of CGT increases with increasing filter width in particular when $\Delta \gg \delta_{t h}$. Furthermore, the extent of CGT decreases with increasing (decreasing) $L e(\tau)$. The effects of CGT diminish with increasing turbulence intensity $u^{\prime} / S_{L}$, which has been demonstrated in Ref. [4].
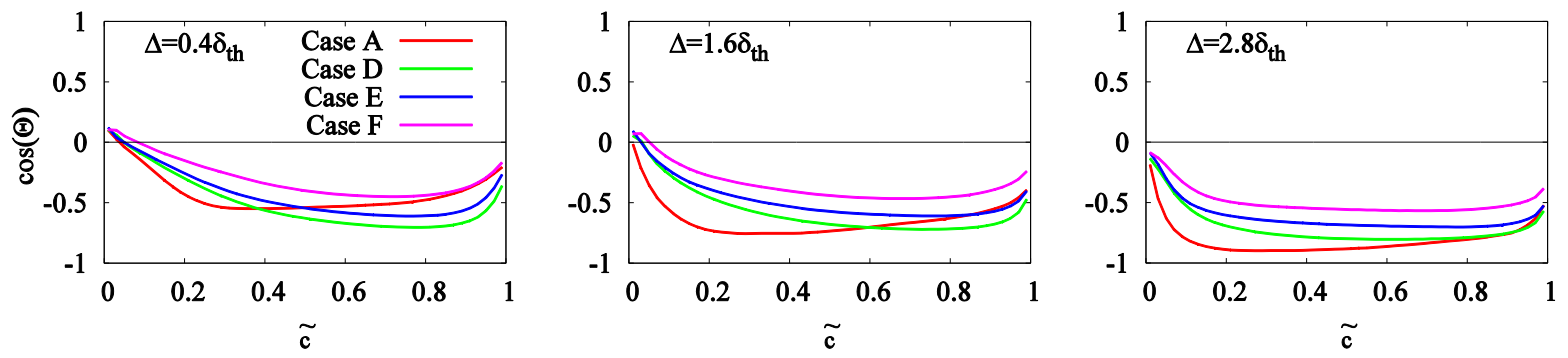

Figure 2: Cosine of the angle $\Theta$ between $\tau_{1 j}^{S G S}$ calculated from DNS and $\tau_{1 j}^{S S M}$ predictions conditional on $\widetilde{c}$ for cases $\mathrm{A}, \mathrm{D}, \mathrm{E}, \mathrm{F}$, for $\Delta \approx 0.4 \delta_{t h}, 1.6 \delta_{t h}$ and $2.8 \delta_{t h}$.

As a consequence of the CGT, a negative correlation can often be observed between eddy viscosity type models and the SGS stresses evaluated from DNS, as shown in Fig. 3. It is important to mention, that CGT is often associated with a negative correlation but both phenomena are not exactly the same. Furthermore Fig. 2 shows only the angle between two vectors but the strength of the flux is lost in this representation. The component $\tau_{23}$ is not shown because this component vanishes in the present configuration. The components $\tau_{12}\left(\tau_{22}\right)$ and $\tau_{13}\left(\tau_{33}\right)$ are statistically identical for this configuration and are therefore grouped together. Figure 3 shows that the correlation coefficient decreases with decreasing (increasing) $L e(\tau)$ (i.e. from case $\mathrm{F}$ to case A), 
and negative correlations are obtained for $L e \ll 1$ (note that a model and the term to be modelled should ideally have a positive correlation as close as possible to 1.0) due to strong dilatation rate effects caused by thermo-diffusive instability. This effect has been explained elsewhere (e.g. in Chakraborty et al. [18]). It was shown elsewhere [4] that an explicit treatment of the isotropic part of $\tau_{i j}^{S G S}$ improves this situation. Before discussing the modelling of $\tau_{k k}^{S G S}$ in detail, a closer look at the anisotropy of $\tau_{i j}^{S G S}$ is necessary. This is due to the fact that the modelling of $\tau_{k k}^{S G S}$ (see last term of Eq. 2) is affected by dilatation effects which are at the same time the root cause for the anisotropy of the stresses.

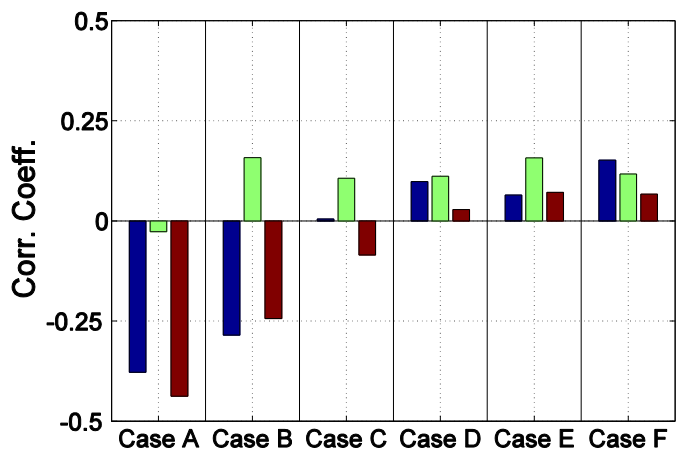

Figure 3: Correlation coefficients for the SSM model for the SGS stress components for cases A-F: $\tau_{11}(\square) ; \tau_{i i, i \neq 1}\left(\right.$ ) and $\tau_{1, i, i \neq 1}(\square)$. Results are averaged over all filter widths.

The anisotropy tensor in the context of LES stresses can be defined as $b_{i j}=\tau_{i j}^{S G S} / \bar{\rho} k^{g s g s}-$ $2 / 3 \delta_{i j}$ where, as noted before, $k^{g s g s}=\tau_{k k}^{S G S} / 2 \bar{\rho}$. The properties of the isotropy tensor can be illustrated looking at the so called Lumley triangle [40] shown in Fig. 4. The boundaries of the triangle can be described in terms of the invariants $I I_{b}=-b_{k k}^{2} / 2$ and $I I I_{b}=-b_{k k}^{3} / 3$ and are given by the expressions $I I I_{b}= \pm 2\left(\left|I I_{b}\right| / 3\right)^{3 / 2}$ where the positive branch corresponds to axisymmetric expansion (see legend in Fig. 4 case F) and the negative part describes axisymmetric contraction. Finally the 2 component state is given by $I I I_{b}=-2 / 3 I I_{b}-8 / 27$. If a coordinate 
system is chosen such that the anisotropy tensor is diagonal, the axisymmetric expansion is given by two equal components of the stress tensor with $b_{22}=b_{33}<0$. Due to the trace free condition this implies that $b_{11}>0$. Further it follows $\tau_{22}, \tau_{33}<2 / 3 \bar{\rho} k^{g s g s}$ and $\tau_{11}>2 / 3 \bar{\rho} k^{g s g s}$.
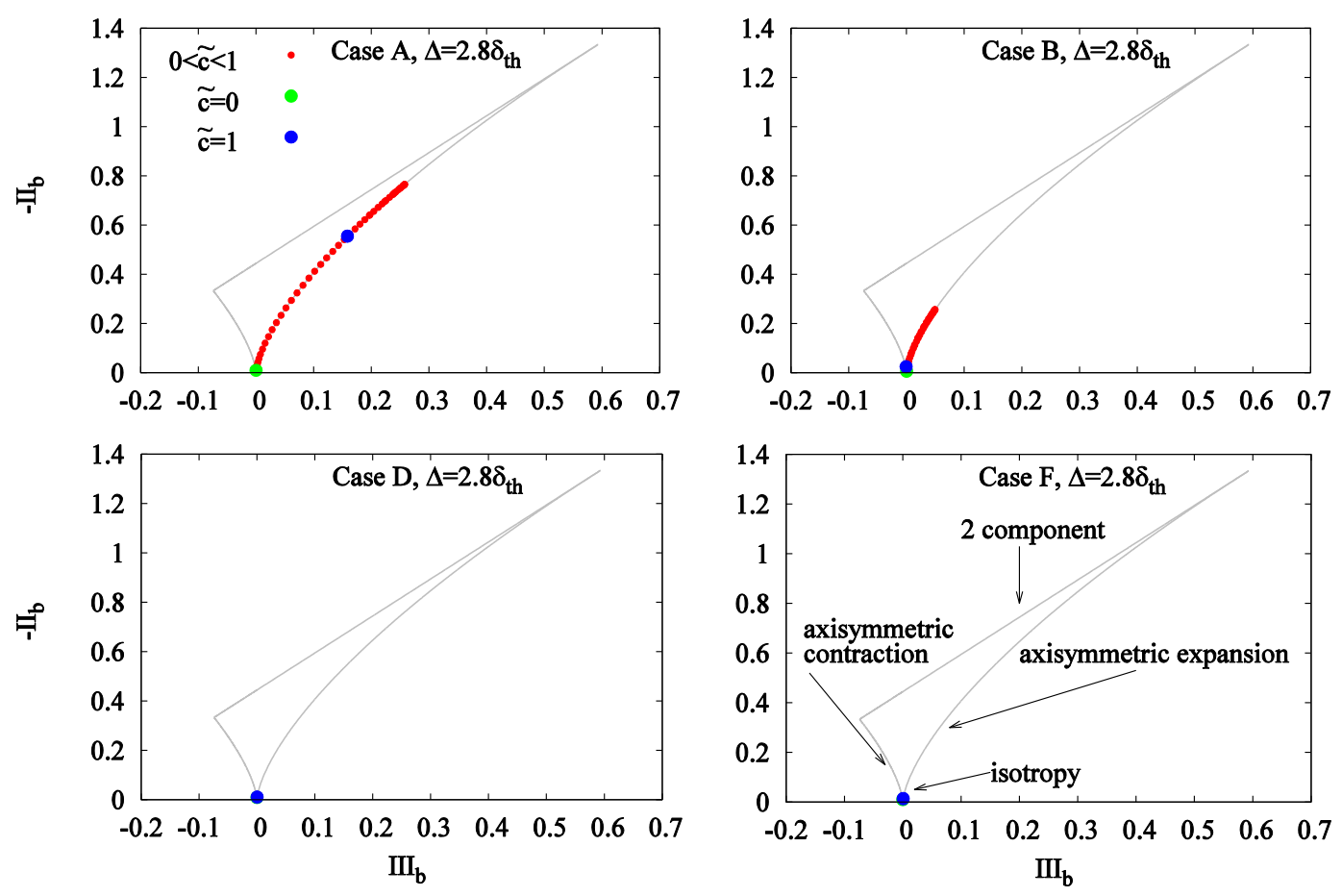

Figure 4: Lumley triangle for cases $A, B, D, F$ for $\Delta=2.8 \delta_{t h}$. Every red dot represents an average conditional on $\tilde{c}$. The points for $\tilde{c}=0$ and $\tilde{c}=1$ are shown in green and blue respectively.

This is exactly the flow state representing a statistically planar flame where mean direction of flame propagation is aligned with the $x_{1}$ axes. The underlying turbulent flow field, without flame, is isotropic and corresponds to the origin of the coordinate system in Fig. 4. Any physical realizable state of the anisotropy tensor $b_{i j}$ has to lie within the triangle. If the invariants of each grid point would be plotted in Fig. 4 the entire triangle would be covered with samples of flow realizations. Simply averaging the coordinates of all realizations will result in points falling outside the triangle because the interior of the triangle is a non-convex set. Therefore the anisotropy tensor has first been averaged conditional on $\tilde{c}$ and then the corresponding invariants have been calculated and 
plotted in the triangle. Each realization in the space of $\tilde{c}$ is shown as a red dot in Fig. 4, whereas $\tilde{c}=0$ and $\tilde{c}=1$ are shown in green and blue colours respectively. Cases A, B, D and F are shown in Fig. 4 because cases $\mathrm{C}$ and $\mathrm{E}$ look qualitatively similar to cases D and F where the flow, at least in an average sense, shows isotropic behaviour. Cases A and $\mathrm{B}$, where $L e \ll 1$, show the strong effects of anisotropy arising from high values of dilatation rate. For case $\mathrm{B}$, i.e. $L e=0.6$, the completely burned and unburned state are in isotropic state but the points corresponding to the flame brush $0<\tilde{c}<1$ are not. For case A $(L e=0.34)$ the burned gas side does not exhibit an isotropic state due to temperature inhomogeneities giving rise to ongoing dilatation effects (see $T$ field for this case in Ref. [19]). Figure 4 illustrates that eddy viscosity type models are expected to fail within the flame brush for cases A and B. Note that the observed isotropy in the other cases is most likely an artefact of the initial isotropic state of turbulence. Therefore stronger anisotropies may exist for other flame configurations.

Figure 4 shows that there is a strong anisotropy within the flame brush which is caused by dilatational effects. These dilatational effects constitute an important contribution to the isotropic part of the SGS stresses in turbulent premixed flames. If the isotropic part of the SGS stresses is not modelled explicitly but added to the filtered pressure the Smagorinsky model assumes for a statistically planar flame, propagating in negative $x_{1}$ direction (i.e. $\partial \widetilde{u_{2}} / \partial x_{2}=\partial \widetilde{u_{3}} / \partial x_{3} \approx 0$ and $\partial \widetilde{u_{1}} / \partial x_{1}>0$ due to heat release) a negative value $\tau_{11}^{S S M}=-\bar{\rho} v_{t} \frac{4}{3} \widetilde{S_{11}}<0$ in contradiction with observations in [4]. This suggests that the modelling of the isotropic part of the SGS stresses is essential for the closure of $\tau_{i j}^{S G S}$ in the context of eddy viscosity type models. Vreman et al. [36] also indicate that the modelling approach of involving a modified pressure is likely to cause problems in the LES of compressible flows because the pressure field is not only needed for the momentum transport but also for the energy conservation equation and in the equation of state 
where the knowledge of absolute pressure is necessary. Finally, $k^{g s g s}$ is often required as a submodel for parametrizing e.g. the SGS effects of turbulence-chemistry interaction.

Figure 5 shows the correlation coefficients between the YOS, CTM and DSS model predictions and the value of $\tau_{k k}^{s g s}$ obtained from explicitly filtered DNS data, where the correlation coefficients for each case are averaged over all values of $\Delta$ considered here. In Fig. 5a gradients of the model expressions are evaluated using finite-differences on the LES grid following [38]. As a general trend, Fig. 5a shows that all correlations increase from case A to F. The correlation coefficients of the DSS model and the CTM model are very similar except for case A (i.e. $L e=0.34$ ) where the density-based version performs better, which is consistent with previous findings [39]. The YOS model has relatively high correlation coefficients because the terms in the YOS model are similar to those in the CTM model. It has been shown in Ref. [39] that the correlation strength in a-priori analysis of turbulent premixed flames depends considerably on the length of the finite-difference stencil. If the correlation coefficients are calculated based on the DNS grid size their magnitude is considerably higher, see Fig 5b, because the scale-similarity type models are based on Taylor expansions and the accuracy (and truncation error) of such a series representation depends on the grid size. 
(a)

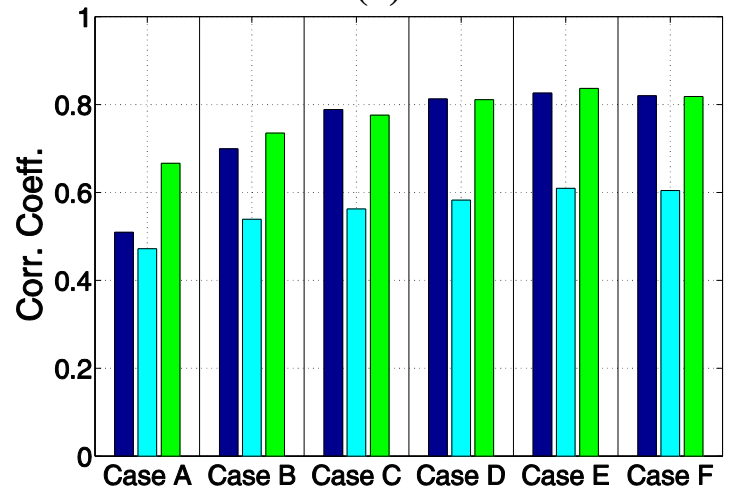

(b)

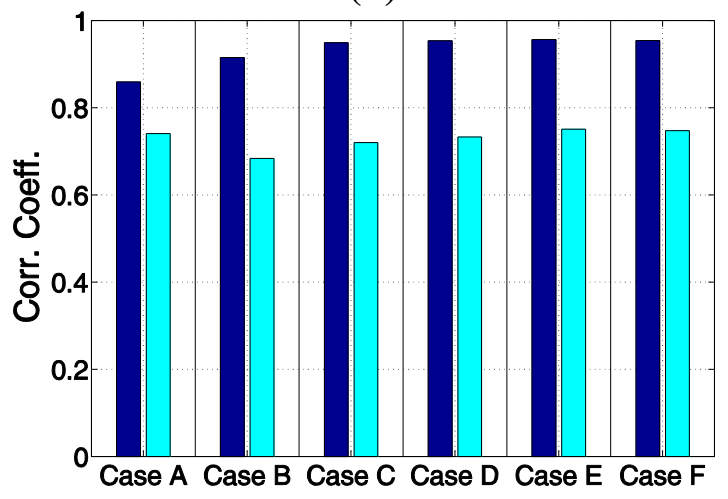

Figure 5: Correlation coefficients between modelled $\tau_{k k}^{s g s}$ and the corresponding value extracted from DNS: CTM $(\square)$; YOS $(\square)$; DSS $(\square)$; (a) Gradients are evaluated on the LES grid. (b) Gradients are evaluated on the DNS grid.

Beside the correlation coefficient, the correct magnitude of the model expression is also important for a successful closure. However, the magnitude of the model expression depends on possible model coefficients, in particular on leading multipliers. The dynamic evaluation of such model multipliers is possible but not a straightforward procedure [4]. Here the calculation is implemented in such a way that the optimal model multiplier is evaluated based on a least-squares fit between the mean values of the model expression and $\tau_{k k}^{s g s}$ evaluated from DNS conditional on $\tilde{c}$.

Figures $6 \mathrm{a}$ and $6 \mathrm{~b}$ show that the optimal model coefficient converges towards unity if $\Delta \rightarrow \Delta_{\mathrm{DNS}}$, which is consistent with the theoretical derivation of the CTM model. Furthermore, the semiempirical choice of $C_{I}=1 / 24$ for the YOS model, is reasonable. It is however unsatisfactory that the optimal model coefficient increases consistently with increasing $\Delta$, and this behaviour is more pronounced going from case $\mathrm{A}$ to case $\mathrm{F}$. 
(a)

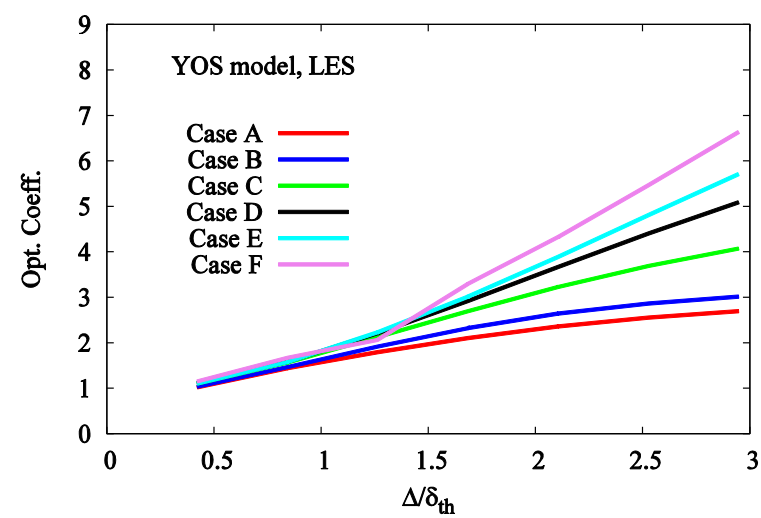

(c)

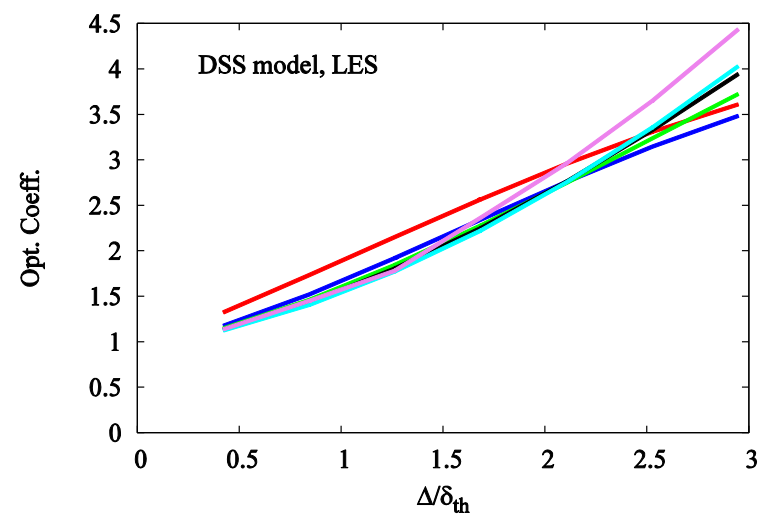

(e)

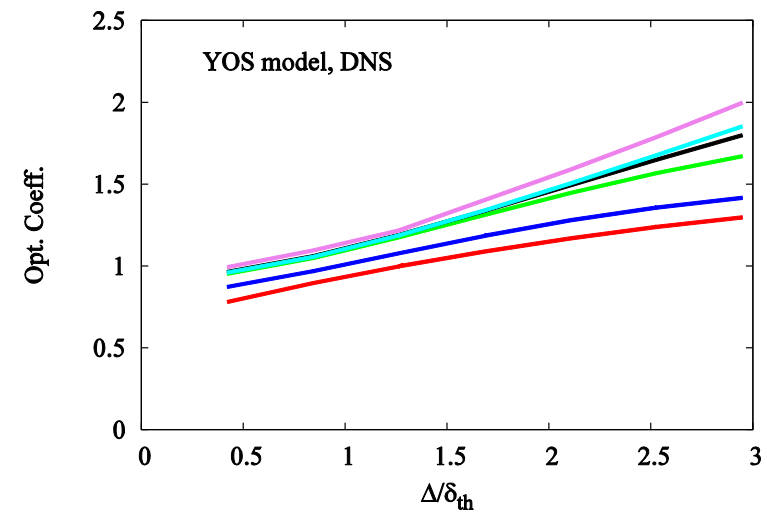

(b)

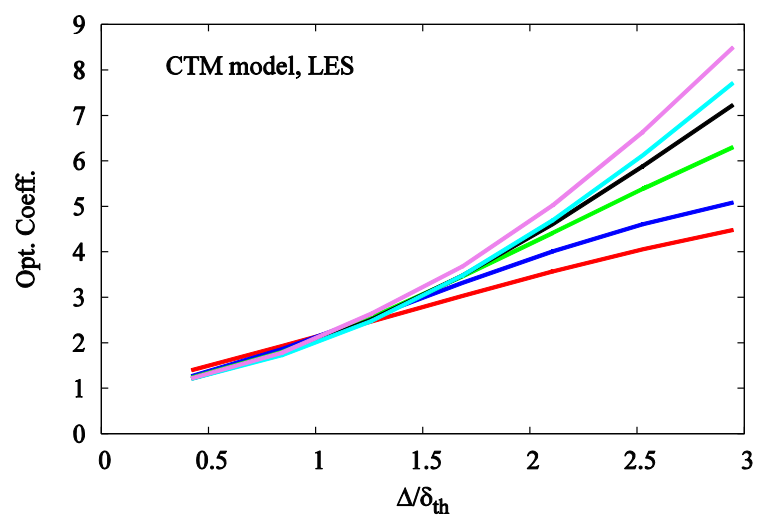

(d)

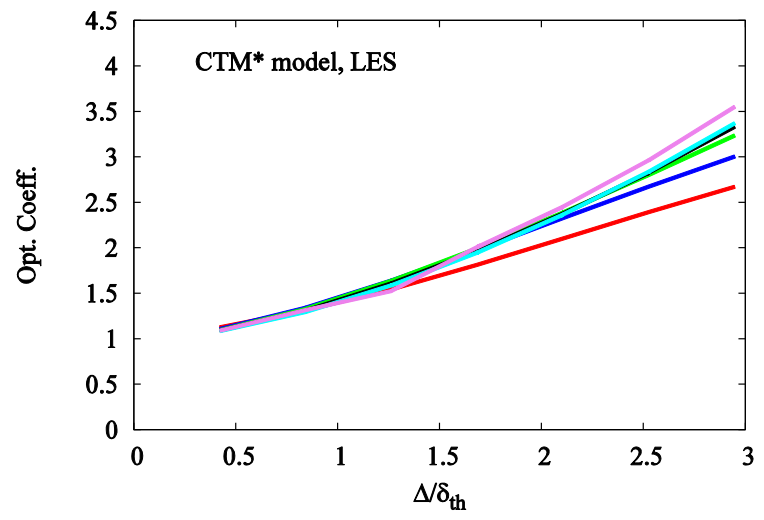

(f)

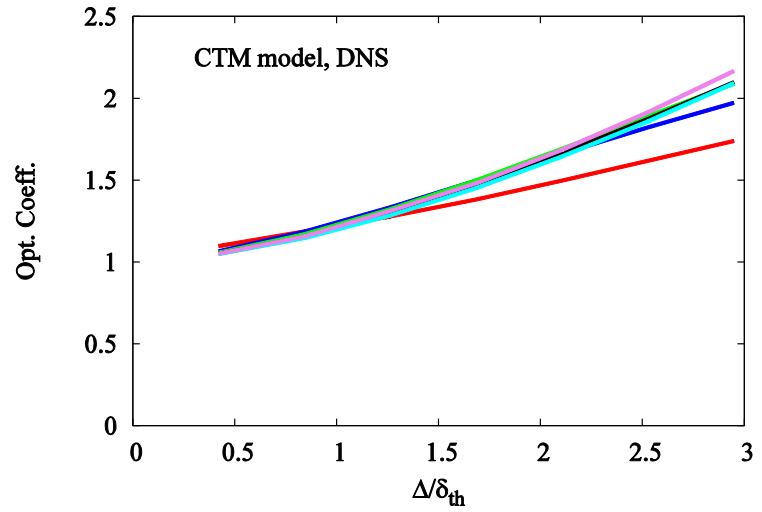

Figure 6: Variation of optimum model multipliers with $\Delta$. (a) YOS and (b) CTM model. Gradients are evaluated on the LES grid. (c) DSS model and (d) CTM* model where gradients are evaluated on the LES grid. (e) YOS and (f) CTM model. Gradients are evaluated on the DNS grid. 
The DSS model shown in Fig. 6c shows exactly the same trend but the model coefficient is considerably more stable with respect to $\Delta$. Since the CTM model is closely related to scalesimilarity type models, the increase in model coefficient with increasing $\Delta$ is likely to be related to the finite-difference operation occurring in the CTM model which is replaced by a filtering in the DSS model. This assumption is further supported by the optimal model coefficients of the $\mathrm{CTM}^{*}$ model in Fig. 6d. Although the CTM* model differs from the CTM model only in its finitedifference approximation, it shows considerably better behaviour in terms of the (under)prediction of the magnitude of $\tau_{k k}^{s g s}$ (equivalent to a model coefficient greater than unity).

Figures 6e and 6f show the predictions of the CTM and YOS models but the finite-differences are approximated on the DNS grid and as a result the model coefficients indeed stay close to unity. A theoretical justification of this behaviour can be given based on the modified wave number [40]. The CTM and YOS models contain products of gradients which are typically evaluated based on central differences. In the $\mathrm{CTM}^{*}$ model the product of this central differences is replaced by a Laplacian. The $2^{\text {nd }}$-order central differencing scheme has a modified wavenumber of $k^{\text {mod }}=$ $\sin (k \Delta x) / \Delta x$. Hence, the product of two first-order derivatives results in a modified squared wavenumber of $\left(k^{\text {mod }}\right)^{2}=(\sin (k \Delta x) / \Delta x)^{2}$, this expression goes to zero very quickly for the highest wavenumbers. In contrast, the modified wavenumber for the $2^{\text {nd }}$ order second derivative (i.e. the Laplacian) has the form $k^{\text {mod }}=4 \sin ^{2}(k \Delta x / 2) / \Delta x^{2}$ which does not approach zero even for the highest wavenumbers. Thus, the CTM* expression is preferred over the CTM expression.

Alternative models for $k^{g s g s}$ could also be obtained by approximating $k^{g s g s}$ by $k^{s g s}=\frac{1}{2} \overline{u_{\Delta l}^{\prime} u_{\Delta l}^{\prime}}$, even though $k^{g s g s} \neq k^{s g s}$. Colin $e t$ al. [12] proposed a model where the dilatation effects are 
removed by applying the rotational operator but this model has been found to be not well-suited when the SGS dilatation effects are strong, e.g. for small Le cases. The correlation coefficients for this model were smaller in the order of 0.1 compared to those of the CTM model in the Le $\approx 1.0$ cases. Another option is to approximate $u_{\Delta}^{\prime}$ as $\|\bar{u}-\overline{\bar{u}}\|$. Correlation coefficients for this approach were found to be 0.3 smaller than those for the CTM model. It was found advantageous to use Favre Filtering, i.e. $u_{\Delta}^{\prime} \approx\|\tilde{u}-\tilde{\tilde{u}}\|$, for case A, where dilatation effects are strong.

\section{CONCLUSIONS}

A-priori assessment of SGS stress closures in premixed turbulent combustion has been conducted using DNS data of statistically planar flames with different $L e$ and $\tau$. Negative correlations between the eddy viscosity type model predictions and SGS stresses extracted from DNS data have been obtained within the flame brush when the isotropic part of the SGS stresses is not modelled. The anisotropy of the SGS stresses has been highlighted using the Lumley triangle. Further, the performance of several models for generalized SGS kinetic energy has been assessed. The main findings are as follows:

1. The CTM model correlates well with DNS data. However, it underpredicts $\tau_{k k}^{s g s}$ for large $\Delta$, and therefore requires an increased model parameter. An alternative formulation, the CTM* model, which is analytically identical, but not numerically equivalent, provides more desirable performance with the variation of $\Delta$.

2. The DSS model shows a slightly better behaviour than the CTM model in terms of model correlation, in particular for small values of Le. Furthermore the model parameter does not change as much as in the case of the CTM model with increasing $\Delta$. 
3. The YOS model shows smaller correlation coefficients than the scale-similarity type models, however the correlation strength remains still reasonable. The similarity between YOS and CTM models was utilized to obtain the semi-empirical value for the model coefficient $C_{I}=1 / 24$. It was shown that this choice yields the correct behavior for small values of $\Delta$. Furthermore, this value satisfies the constraint implied by the realizability conditions put forward in Vreman et al. [36]. The value of the model constant $C_{I}=1 / 24$ is within the large range of values $\left(C_{I}=0, \ldots, 0.089\right)$ recommended in literature $[3,5,6,7]$, but more towards the higher side.

The present findings indicate the strong interaction between the numerics and physical modelling in LES. An optimum parametrization of the model coefficients, by taking into account combined numerical and physical aspects, needs to be addressed in future.

\section{APPENDIX}

\section{A1. Effects of $R e_{t}$ and $l / \delta_{t h}$ on the modelling of GSGS TKE}

Filtering of large three-dimensional datasets for large filter widths is computationally very demanding. For the current analysis moderate values of turbulent Reynolds number and the ratio of integral length scale to flame thickness have been considered for a detailed parametric analysis involving 6 different flames and 7 different filter widths. In order to demonstrate that all findings reported in this work remain valid for higher values of $R e_{t}$ and $l / \delta_{t h}$, a simulation involving unity Lewis number has been carried out for a domain of size $32.3 \delta_{t h} \times 32.3 \delta_{t h} \times 32.3 \delta_{t h}$, which was discretised using a uniform Cartesian mesh of $768^{3}$ grid points. The heat release parameter $\tau$ is taken to be 4.5 , whereas $\operatorname{Pr}, \beta_{Z}$ and $\gamma_{g}$ values are same as cases A-F. The initial values of $u^{\prime} / S_{L}$

and $l / \delta_{t h}$ for this additional case are taken to be 15.0 and 5.72. The initial values of turbulent 
Reynolds, Damköhler and Karlovitz numbers are $R e_{t}=216, D a=0.38$ and $K a=24.28$ respectively. This DNS data has been explicitly filtered using a Gaussian filter kernel ranging from $\Delta \approx 0.3 \delta_{t h}$ where the flame is almost resolved, up to $\Delta \approx 4.2 \delta_{t h}$ where the flame becomes fully unresolved and $\Delta$ is comparable to the integral length scale $l$. It is worth noting that the scale separation $l / \delta_{t h}$ and the turbulent Reynolds number are considerably larger than the Lewis number database discussed in this work. Nevertheless results are perfectly in line with the findings reported earlier. Exemplarily, the cosine of the angle $\Theta$ between $\tau_{1 j}^{S G S}$ calculated from DNS and $\tau_{1 j}^{S S M}$ predictions conditional on $\widetilde{c}$ is at the order of 0.1-0.2 smaller compared to the results shown in Fig. 2, but still negative and qualitatively similar. Further, the amount of CGT increases with increasing filter width. The correlation coefficients averaged over all filter widths for the SSM model for the SGS stress components are $\tau_{11}=-0.07, \tau_{i i, i \neq 1}=0.18$, and $\tau_{1, i, i \neq 1}=0.08$. The order of magnitude is similar to the unity Lewis number case and also the relative magnitude of the different stresses shows the same behaviour as depicted in Fig. 2, in the sense that $\tau_{22}, \tau_{33}$ have the highest positive correlations whereas the stress components involving the direction of mean flame propagation are considerably smaller or even negative. Finally, the optimal model multipliers for $\Delta / \delta_{t h}=0.3,1.2,2.1,4.2$ (gradients are evaluated on the LES grid) are given by $1.07,2.02,3.18,5.21$ for the YOS model and $1.12,2.17,3.57,6.52$ for the CTM model respectively. In the context of the SGS-stress closure, the appropriate parameter for comparing the results for this new case with cases A-F is is $\Delta / l$ rather than $\Delta / \delta_{t h}$. A comparison between the above numbers and the corresponding values reported in Fig. 6 reveals a very good qualitative and quantitative agreement. 


\section{A2. Positivity of $\tau_{k k}^{C T M *}$ for arbitrary discrete velocity fields}

The positivity of the discretized expression for $\tau_{k k}^{C T M *}$ will be discussed in this section. Obviously, for a consistent discretization scheme the quantity will be positive in the limit of small grid size, because we are approximating a differential operator that can only assume positive values. It will be difficult to discuss positivity for any arbitrary discretization scheme of the second derivative on arbitrary grids. Instead let us consider two particular examples:

Without loss of generality we consider the (one-dimensional) expression $\bar{\rho}\left(\Delta^{2} / 24\right)\left(\partial^{2}\left(u^{2}\right) / \partial x^{2}-2 u \partial^{2} u / \partial x^{2}\right)$. Let us write $u_{e}, u_{p}, u_{w}$ instead of $u(i+1), u(i), u(i-$ 1) respectively. We approximate $\bar{\rho}\left(\Delta^{2} / 24\right)\left(\partial^{2}\left(u^{2}\right) / \partial x^{2}-2 u \partial^{2} u / \partial x^{2}\right)$ by a second order accurate finite difference (which was the focus in this work) for the second derivative. This gives

$$
\begin{gathered}
\bar{\rho}\left(\Delta^{2} / 24\right)\left(\partial^{2}\left(u^{2}\right) / \partial x^{2}-2 u \partial^{2} u / \partial x^{2}\right) \approx \\
\bar{\rho}\left(\Delta^{2} / 24\right) / \Delta x^{2}\left[\left(u_{e}^{2}-2 u_{p}^{2}+u_{w}^{2}\right)-2 u_{p}\left(u_{e}-2 u_{p}+u_{w}\right)\right]
\end{gathered}
$$

and it will be sufficient to show $\left(u_{e}^{2}-2 u_{p}^{2}+u_{w}^{2}\right)-2 u_{p}\left(u_{e}-2 u_{p}+u_{w}\right)>0$ which is in fact easy to demonstrate:

$$
\begin{aligned}
\left(u_{e}^{2}-2 u_{p}^{2}+u_{w}^{2}\right)-2 u_{p}\left(u_{e}-2 u_{p}+u_{w}\right)=u_{e}^{2}+u_{w}^{2}-2 u_{p} u_{e}+2 u_{p}^{2}-2 u_{p} u_{w} \\
=u_{e}^{2}-2 u_{p} u_{e}+u_{p}^{2}+u_{w}^{2}-2 u_{p} u_{w}+u_{p}^{2}=\left(u_{e}-u_{p}\right)^{2}+\left(u_{w}-u_{p}\right)^{2}>0
\end{aligned}
$$

Hence we note that for a second order accurate standard discretization the expression will be positive on equidistant grids.

Let us next consider the fourth order accurate finite difference of the second derivative, i.e.

$$
\begin{gathered}
\bar{\rho}\left(\Delta^{2} / 24\right)\left(\partial^{2}\left(u^{2}\right) / \partial x^{2}-2 u \partial^{2} u / \partial x^{2}\right) \approx \\
\bar{\rho}\left(\Delta^{2} / 24\right) /\left(12 \Delta x^{2}\right)\left[\left(-u_{e e}^{2}+16 u_{e}^{2}-30 u_{p}^{2}+16 u_{w}^{2}-u_{w w}^{2}\right)\right. \\
\left.-2 u_{p}\left(-u_{e e}+16 u_{e}-30 u_{p}+16 u_{w}-u_{w w}\right)\right]
\end{gathered}
$$


In this context it is instructive to analyse the expression:

$$
\begin{gathered}
{\left[\left(-u_{e e}^{2}+16 u_{e}^{2}-30 u_{p}^{2}+16 u_{w}^{2}-u_{w w}^{2}\right)-2 u_{p}\left(-u_{e e}+16 u_{e}-30 u_{p}+16 u_{w}-u_{w w}\right)\right]} \\
=16\left(u_{e}-u_{p}\right)^{2}+16\left(u_{w}-u_{p}\right)^{2}-\left(u_{w w}-u_{p}\right)^{2}-\left(u_{e e}-u_{p}\right)^{2}
\end{gathered}
$$

This expression will not be positive for arbitrary discrete velocity fields, e.g. $u_{e}=u_{p}=u_{w}=$ $u_{w w}=0, u_{e e} \neq 0$. Looking only at the east part of this symmetric expression it can be seen that

$16\left(u_{e}-u_{p}\right)^{2}-\left(u_{e e}-u_{p}\right)^{2}<0$, if $\left(u_{e e}-u_{e}\right)>3\left(u_{e}-u_{p}\right)$. This situation can occur if the velocity field is not smooth enough, i.e. if it is not very well resolved. Applying this formula to a simulated turbulent, reasonably resolved velocity field indicated that very seldom negative values occur. However, if negative values occur, their magnitude is very small and they can safely be clipped to zero.

\section{ACKNOWLEDGEMENTS}

NC is grateful to EPSRC, UK and ARCHER for financial and computational assistance respectively. 


\section{REFERENCES}

[1] J. Chomiak and J. Nisbet, Modeling variable density effects in turbulent flames - some basic considerations, Combustion and Flame, 102, (1995), 371-386.

[2] P. Pfadler, F. Beyrau, F. Dinkelacker and A. Leipertz, A-priori testing of an eddy viscosity model for the density-weighted subgrid scale stress tensor in turbulent premixed flames, Experiments in Fluids. 49, (2010), 839-851.

[3] E. Garnier, P. Sagaut and N. Adams, Large Eddy Simulation for Compressible Flows, Springer (2009).

[4] M. Klein, C. Kasten, Y. Gao and N. Chakraborty, A-priori direct numerical simulation assessment of sub-grid scale tensor closures for turbulent premixed combustion. Computers and Fluids, 122, (2015) 1-11.

[5] A. Yoshizawa, Statistical theory for compressible turbulent shear flows, with the application to subgrid modeling, Physics of Fluids, 29, (1986), 2152-2164.

[6] P. Moin, K. Squires, W. Cabot and S. Lee, A dynamic subgrid scale model for compressible turbulence and scalar transport, Physics of Fluids, A 3, (1991), 2746-2757.

[7] T.A. Zang, R.B. Dahlburg and J.P. Dahlburg, Direct and large eddy simulations of threedimensional compressible Navier-Stokes turbulence, Physics of Fluids, 4, (1992), 127-140.

[8] Y. Gao, N. Chakraborty and M. Klein, Assessment of the performances of sub-grid scalar flux models for premixed flames with different global lewis numbers: A direct numerical simulation analysis, International Journal of Heat and Fluid Flow, 52, (2015), 28-39.

[9] Y. Gao, M. Klein and N. Chakraborty, Assessment of sub-grid scalar flux modelling in premixed flames for large eddy simulations, European Journal of Mechanics - B/Fluids, 52, (2015), $97-108$. 
[10] M. Germano, Turbulence: the filtering approach, Journal of Fluid Mechanics, 238, (1992), 325-336.

[11] H. Pitsch, and H. Duchamp de Lageneste, Large-Eddy Simulation of premixed turbulent combustion using a level-set approach, Proceedings of the Combustion Institute, 29, (2002), 20012008.

[12] O. Colin, F. Ducros, D. Veynante and T. Poinsot, A thickened flame model for large eddy simulations of turbulent premixed combustion, Phys. Fluids, 12(7), (2000), 1843-1863.

[13] C. Fureby, A fractal frame-wrinkling Large Eddy Simulation model for premixed trubulent combustion, Proceedings of the Combustion Institute, 30, (2005), 593-601.

[14] N. Chakraborty and M. Klein, A-priori numerical simulation assessment of algebraic flame surface density models for turbulent premixed flames in the context of large eddy simulation, Physics of Fluids, 20, (2008), 085108.

[15] R. Keppeler, E. Tangermann, U. Allaudin, M. Pfitzner, LES of Low to High Turbulent Combustion in an Elevated Pressure Environment, Flow, Turbulence and Combustion, 92, (2014), 767-802.

[16] M. Klein, An attempt to assess the quality of large eddy simulations in the context of implicit filtering, Flow, Turbulence and Combustion, 75, (2005), 131-147.

[17] N. Chakraborty, R.S. Cant, Effects of Lewis number on turbulent scalar transport and its modelling in turbulent premixed flames, Combustion and Flame. 156, (2009), 1427-1444.

[18] N. Chakraborty, M. Klein, N. Swaminathan, Effects of Lewis number on the reactive scalar gradient alignment with local strain rate in turbulent premixed flames. Proceedings of the Combustion Institute, 32, (2009), 1409-1417. 
[19] N. Chakraborty, and R.S. Cant, Effects of Lewis number on flame surface density transport in turbulent premixed combustion, Combustion and Flame, 158, (2011), 1768-1787.

[20] N. Chakraborty, M. Katragadda, R.S. Cant, Effects of Lewis number on turbulent kinetic energy transport in premixed flames, Physics of Fluids, 23, (2011) 075109.

[21] N. Chakraborty, A.N. Lipatnikov, Effects of Lewis number on conditional fluid velocity statistics in low Damköhler number turbulent premixed combustion: A direct numerical simulation analysis, Physics of Fluids, 25, (2013), 045101.

[22] M. Katragadda, N. Chakraborty and R.S. Cant, A Priori Assessment of Algebraic Flame Surface Density Models in the Context of Large Eddy Simulation for Nonunity Lewis Number Flames in the Thin Reaction Zones Regime, Journal of Combustion, (2012), 794671.

[23] Y. Gao, N. Chakraborty and N. Swaminathan, Algebraic Closure of Scalar Dissipation Rate for large Eddy Simulations of Turbulent Premixed Combustion, Combustion Science and Technology, 186, (2014) 1309-1337.

[24] N. Peters, Turbulent Combustion. Cambridge University Press, UK (2000).

[25] M. Boger, D.Veynante, H. Boughanem, and A. Trouvé, Direct numerical simulation analysis of flame surface density concept for large eddy simulation of turbulent premixed combustion, Proceedings of the Combustion Institute, 27, (1998) 917-925.

[26] O. Colin, F. Ducros, D. Veynante, T. Poinsot, A thickened model for large eddy simulations of turbulent premixed combustion, Physics of Fluids, 12(7), (2000), 1843-1863.

[27] F. Charlette, C. Meneveau, D. Veynante, A power-law flame wrinkling model for LES of premixed turbulent combustion Part I: non-dynamic formulation and initial tests, Combustion and Flame, 131, (2002), 159-180. 
[28] I. Han, K.H. Huh, Roles of displacement speed on evolution of flame surface density for different turbulent intensities and Lewis numbers in turbulent premixed combustion. Combustion and Flame, 152, (2008), 194-205.

[29] H. Reddy and J. Abraham, Two-dimensional direct numerical simulation of the flame surface density model for flames developing from an ignition kernel in lean methan/air mixtures under engine conditions, Physics of Fluids, 24, (2012), 105108.

[30] T. Ma, T.O. Stein, N. Chakraborty, A.M. Kempf, A posteriori testing of algebraic flame surface density models for LES, Combust. Theor. and Modell. 17, (2013) 431-482.

[31] T. Ma, Y. Gao, A.M. Kempf, N. Chakraborty, Validation and implementation of algebraic LES modelling of scalar dissipation rate for reaction rate closure in turbulent premixed combustion. Combustion and Flame, 161,12 (2014) 3134-3153.

[32] C. Dopazo, L. Cifuentes, J. Martin, C. Jimenez, Local flow topologies and scalar structures in a turbulent premixed flame, Physics of Fluids, 26 (2014) 065108.

[33] C. Dopazo, L. Cifuentes, J. Martin, C. Jimenez, Stain rates normal to approaching iso-scalar surfaces in a turbulent premixed flame, Combustion and Flame, 162, (2014) 1729-1736.

[34] R.A. Clark, J.H. Ferziger and W.C. Reynolds, Evaluation of subgrid-scale models using an accurately simulated turbulent flow, Journal of Fluid Mechanics, 91, (1979) 1-16.

[35] B. Vreman, Direct and Large Eddy Simulation of the Compressible Mixing Layer, PhD Thesis, University of Twente, (1995).

[36] B. Vreman, B. Geurts and H. Kuerten, Realizability conditions for the turbulent stress tensor in large-eddy simulations, J. Fluid Mech. 278, (1994), 351-362.

[37] B.W. Anderson and J.A. Domaradzki, A subgrid-scale model for LES Based on the physics of interscale energy transfer turbulence, A Phys. Fluids, 24, (2012), 065104. 
[38] S. Liu, C. Meneveau and J. Katz, On the properties of similarity subgrid-scale models as

deduced from measurements in a turbulent jet, Journal of Fluid Mechanics, 275, (1994), 83-119.

[39] M. Klein, N. Chakraborty and Y. Gao, Scale similarity based models and their application to subgrid scale scalar flux modelling in the context of turbulent premixed flames, International Journal of Heat and Fluid Flow, 57, (2015), 91-108.

[40] P.A. Durbin, B. A. Pettersson Reif, Statistical Theory and Modeling for Turbulent Flows, John Wiley and Sons, New York, NY, USA (2001). 


\section{FIGURE CAPTIONS}

Figure 1: Instantaneous view of $c$ isosurfaces for cases A and D at $t=\delta_{t h} / S_{L}$. The value of $c$ increases from 0.1 (yellow) to 0.9 (red).

Figure 2: Cosine of the angle $\Theta$ between $\tau_{1 j}^{S G S}$ calculated from DNS and $\tau_{1 j}^{S S M}$ predictions conditional on $\widetilde{c}$ for cases A,D,E,F, for $\Delta \approx 0.4 \delta_{t h}, 1.6 \delta_{t h}$ and $2.8 \delta_{t h}$.

Figure 3: Correlation coefficients for the SSM model for the SGS stress components for cases AF: $\tau_{11}(\square) ; \tau_{i i, i \neq 1}(\square)$ and $\tau_{1, i, i \neq 1}(\square)$. Results are averaged over all filter widths.

Figure 4: Lumley triangle for cases A,B,D,F for $\Delta=2.8 \delta_{t h}$. Every red dot represents an average conditional on $\tilde{c}$. The points for $\tilde{c}=0$ and $\tilde{c}=1$ are shown in green and blue respectively.

Figure 5: Correlation coefficients between modelled $\tau_{k k}^{s g s}$ and the corresponding value extracted from DNS: CTM ( $\square$ ); YOS $(\square)$; DSS ( $\square$ ); (a) Gradients are evaluated on the LES grid. (b) Gradients are evaluated on the DNS grid.

Figure 6: Variation of optimum model multipliers with $\Delta$. (a) YOS and (b) CTM model. Gradients are evaluated on the LES grid. (c) DSS model and (d) CTM* model where gradients are evaluated on the LES grid. (e) YOS and (f) CTM model. Gradients are evaluated on the DNS grid. 